\title{
SECRETARÍA DE INVESTIGACIÓN Y VINCULACIÓN INTERNACIO- NAL DE LA FACULTAD DE DERECHO
}

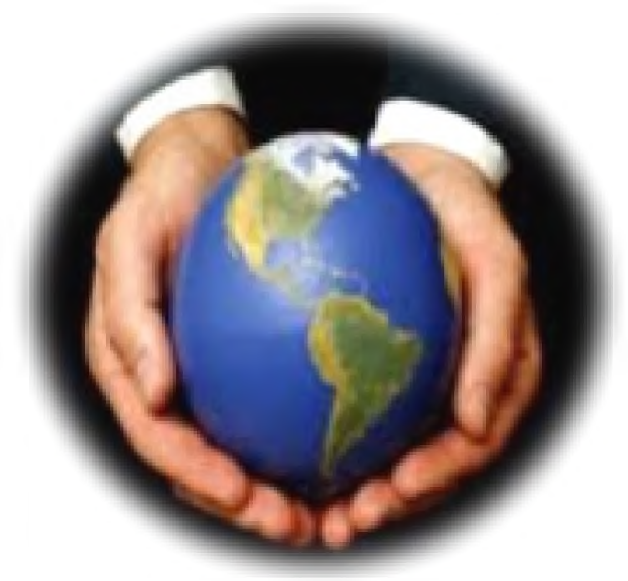

En la Facultad de Derecho y Ciencias Sociales y Políticas de la UNNE, La Secretaría de Investigación y Vinculación Internacional ha venido en los últimos tiempos ejecutando importantes políticas activas destinadas a la creación y perfeccionamiento de sus docentes con el Programa Formación Docente Continua en Investigación, alentando y promoviendo acceso a la formación de los docentes y auxiliares docentes de la Facultad, investigadores y becarios a fin de incentivar el perfeccionamiento académico de su planta docente.

Asimismo, es parte de esta política de desarrollo el fomento constante del intercambio internacional entre estudiantes, docentes e investigadores de ésta y otras Casas de Altos Estudios de la Argentina, el MERCOSUR y Latinoamérica y Europa en general, propiciando la incorporación de los avances pedagógicos de los sistemas educativos de otros países en la formación profesional de los docentes, facilitando el intercambio de experiencias mediante la práctica docente en otros sistemas educativos, estimulando la ejecución de investigaciones y proyectos educativos en áreas específicas del trabajo docente con el objetivo de aportar posibles soluciones a problemas comunes y con el fin último de lograr la construcción de un sistema educativo ajustado a las exigencias académicas que demandan los tiempos que corren. Se detallan a continuación algunas de las actividades más destacadas de la Secretaria: 


\section{Actividades}

\section{Jornada de Becarios de la Facultad de Derecho}

Con el doble objetivo de incentivar la excelencia en el rendimiento académico de los alumnos de grado y promover masiva y abiertamente las actividades de intercambio internacional e investigación desarrolladas por nuestra Universidad, se ha llevado a cabo en el Campus de la Facultad de Derecho la V Jornada de Becarios, que ha contado con la activa participación de la Rectora de la Universidad Nacional del Nordeste: Prof. María Delfina Veiravé; Secretaria General de Ciencia y Técnica - UNNE - : Dra. María Silvia Leoni y el Secretario General de Relaciones Interinstitucionales - UNNE-: Arq. Gustavo Alejandro Tripaldi, además de la presencia de otras destacadas autoridades del ámbito académico.

En el marco de esta actividad, los becarios de intercambio internacional y de investigación de esta Unidad Académica han compartido generosamente con un numeroso público de alumnos e interesados sus experiencias y vivencias personales vinculadas a este reconocimiento académico de los méritos logrados en el curso de la Carrera de Grado.

\section{Becas de intercambio estudiantil}

Desde la convocatoria del año 2008 al $1^{\circ}$ semestre del año 2015, la Facultad de Derecho ha sido adjudicada en un total de 34 becas de intercambio a los países que a continuación se detallan:

\begin{tabular}{|c|c|c|c|c|c|}
\hline México & Paraguay & Brasil & Bolivia & Chile & Colombia \\
\hline 14 & 5 & 15 & 3 & 1 & 5 \\
\hline
\end{tabular}

\section{Estudiantes extranjeros}

$1^{\circ}$ y $2^{\circ}$ Semestre 2016: (10 estudiantes Extranjeros)

- William García Reyes (Universidad de Magdalena-Colombia).

- Krisstarah Dayana Lobo (Universidad Federal de Santa Catarina-Brasil).

- Cipriani de Moura Brena (Universidad Federal de Santa María-Brasil).

- Hermosilla Ferreira Walter (Universidad Nacional de Itapúa-Paraguay). 
- Alves Carvalho Gabriel Julio (Universidad Federal de Rio Grande do Sul).

- Jair Abel Martínez Chávez (Universidad de Guadalajara-México).

- Becerra Cusi Andrea (Universidad de Sonora-México).

- Quijada Marciel Carlos Alberto (Universidad de Sonora-México).

- Solorzano Cota Héctor Gastón (Universidad de Sonora-México).

- Zazueta Martínez Narce Alejandra (Universidad de Sonora-México).

Estudiantes de la facultad que realizaron movilidad durante el año 2016 Programa JIMA

\begin{tabular}{|c|c|}
\hline Nombre & Destino \\
\hline Stella Maris Gimenez & Universidad Veracruzana \\
\hline
\end{tabular}

Programa MACA

\begin{tabular}{|c|c|}
\hline Nombre & Destino \\
\hline Britos, Maria Florencia & Universidad del Valle \\
\hline
\end{tabular}

Programa escala estudiantil

\begin{tabular}{|c|c|}
\hline Nombre & Destino \\
\hline Gonzalez Sasson, Andrea Elien & $\begin{array}{c}\text { Universidad Federal de San } \\
\text { Carlos - Brasil (UFSCAR) }\end{array}$ \\
\hline Vergara, Paola Marianela & $\begin{array}{c}\text { Universidad Nacional de } \\
\text { Asunción - Paraguay (UNA) }\end{array}$ \\
\hline Majul Maria Luz & Universidad de Campinas \\
\hline Atanasoff Cristosoff Carolina & $\begin{array}{c}\text { Universidad San Francisco Xavier } \\
\text { de Chuquisaca }\end{array}$ \\
\hline
\end{tabular}




\section{Becas de Investigación}

Durante la convocatoria 2016 realizada por la Secretaria de Ciencia y Técnica de la Universidad Nacional del Nordeste, se han otorgado las siguientes Becas de Investigación:

- Nueve (9) Becas de Pregrado.

- Dos (2) Becas de Iniciación.

- Una (1) Becas de Perfeccionamiento.

\section{Grupos de investigación y Proyectos acreditados}

La Facultad de Derecho tiene aprobados a la fecha un total de 18 grupos de investigación y 25 proyectos acreditados.

\section{Convenios marcos y específicos. Acuerdos y actas firmados y en trámite}

- De Colaboración y Cooperación Académica entre la Universitat Pompeu Fabra (España) y la Universidad Nacional del Nordeste. (En trámite).

- Convenio Marco entre La Universitat Pompeu Fabra (España) y la Universidad Nacional del Nordeste. (En trámite).

- Convenio de Cooperación y Asistencia entre la Facultad de Derecho y Ciencias Sociales y Políticas y la Federación de Cooperativas de Corrientes LTDA. (En trámite).

- Asociación Civil de Abogados Previsionalistas (Acap).

- Acuerdo Específico de Colaboración y Cooperación Académica entre la Facultad de Derecho y Ciencias Sociales y Políticas y la Facultad de Ciencias Económicas, ambas dependientes de la Universidad Nacional del Nordeste.

- Entre la Facultad de Derecho de Ciencias Políticas y Sociales de la Universidad Nacional del Nordeste y el Instituto Argentino de la Empresa Familiar Asociación Civil (IADEF).

- Acta intención de celebración de convenio entre la Facultad de Derecho de Ciencias Sociales y Políticas de la Universidad Nacional del Nordeste y la Facultad de Derecho de Università Degli Studi Di Messina (Italia). 
Programa formación docente continua en investigación aprobado por Res. 219 C.D. $/ 14$

Durante el año 2016 se han llevado a cabo los siguientes cursos y talleres:

1) Taller: "Escribir para publicar desde un enfoque situado".

2) Taller de elaboración de proyectos de investigación y escritura científica.

Jornadas y talleres organizados por la Secretaría de Investigación y Vinculación Internacional

1) Jornada en Homenaje al Bicentenario de la Independencia (1816-2016): "Ideas políticas y contribuciones institucionales en el proceso independentista argentino".

Programa formación continua para becarios aprobado por Res. 458 C.D./14

Durante el año 2016 se han llevado a cabo los siguientes cursos y talleres:

1) V Jornada de becarios de la Facultad de Derecho de la UNNE (Abril 2016).

2) Primer taller de formación continua para becarios: "Derechos y obligaciones de los becarios" (Marzo 2016).

3) Segundo taller de formación continua para becarios: "Trabajando el C.V. de un becario" (Abril 2016).

4) Tercer taller de formación continua para becarios: "Escritura de comunicaciones y artículos científicos" (Mayo 2016).

\section{Comunicaciones científicas}

En las $\mathrm{XII}^{\circ}$ Jornadas de Comunicaciones Científicas y $\mathrm{II}^{\circ}$ Internacional llevada a cabo durante el año 2016 se han presentado ciento veinte 120 trabajos de Investigadores, y se ha realizado la presentación del Libro de las $\mathrm{XI}^{\circ}$ Jornadas de Comunicación Científicas de la Facultad de Derecho. 